\title{
The Effect of a Frequency-Doubled Q-Switched Nd:YAG Laser on Hairless Mice Harboring Eumelanin and Pheomelanin in the Epidermis
}

\author{
Hanao Yamaguchi, Yoko Funasaka and Hidehisa Saeki \\ Department of Dermatology, Nippon Medical School, Tokyo, Japan
}

Background: For laser therapy, darker skin types should be carefully treated, however, the precise role of melanin content, subspecies, and the heat effect of the laser has not been well studied in vivo.

Methods: We generated three groups of mice that have epidermal melanocytes producing only eumelanin, dominant pheomelanin, and no melanin. Using these mice, the effect of a frequency-doubled Nd: YAG laser was studied.

Results: The mouse epidermis that contained eumelanin underwent heat degeneration at a lower fluence when compared with the mouse epidermis with dominant pheomelanin. The mouse skin with no melanin did not show any degeneration of the epidermis.

Conclusion: The effect of the Nd:YAG laser on the cells containing different melanin subspecies was shown to be different in an in vivo irradiation system. (J Nippon Med Sch 2019; 86: 27-31)

Key words: Nd:YAG laser, eumelanin, pheomelanin

\section{Introduction}

Constitutive pigmentation of human skin is determined by melanin content as melanocytes produce two chemically distinct types of melanin: the insoluble black to brown eumelanin, and the alkaline-soluble, yellow to reddish-brown pheomelanin. Both eumelanin and pheomelanin are derived from the common precursor dopaquinone, however, the availability of cysteine leads to formation of the pheomelanin pigment. Cysteine reacts rapidly and quantitatively with dopaquinone to produce 5-cysteinyl-dopa (5SCD) and 2-S-cysteinyldopa (2SCD) ${ }^{1}$. Cysteinyldopas are then oxidized by dopaquinone to give benzothiazine intermediates, which gradually polymerize to form pheomelanin pigments. In the late stage of pheomelanin production, the benzothiazine moiety is gradually converted to a benzothiazole moiety ${ }^{2}$. When cysteine is depleted in melanosomes, dopaquinone spontaneously reacts to give 5,6-dihydroxyindole (DHI) and 5,6-dihydroxyindole-2-carboxylic acid (DHICA) via dopachrome. DHICA formation is also accelerated by dopachrome tautomerase. These dihydroxyindoles are then further oxidized to produce the eumelanin polymer $^{1}$.
Various lasers were developed to destroy specifically melanin-containing organelles or cells. To evaluate this, the skin specimens were taken and evaluated by observing melanosome injury as well as temporary whitening of the skin due to microcavity formation by heat. The optical absorption of melanin has been well studied using extracted or synthetic melanin to determine the proper fluence of a laser to heat up melanin ${ }^{3}$. However, there is no in vivo study of injury of melanin-containing organelles or cells by laser irradiation with the information of the amount of each species of melanin in the skin. We have previously generated hairless mice harboring epidermal melanocytes producing dominantly eumelanin, pheomelanin, or no-melanin and elucidated the content of each type of melanin in the epidermis ${ }^{4}$. Using these mice, we examined the photothermal effect of a frequency-doubled Q-switched Nd:YAG laser on the pigmentary epidermis in our current study. This study might offer a more detailed explanation for the relationship between the amount of melanin and the injury of melanin-containing cells.

Correspondence to Yoko Funasaka, Department of Dermatology, Nippon Medical School, 1-1-5 Sendagi, Bunkyo-ku, Tokyo 113-8603, Japan

E-mail: funasaka@nms.ac.jp

Journal Website (http://www2.nms.ac.jp/jnms/) 


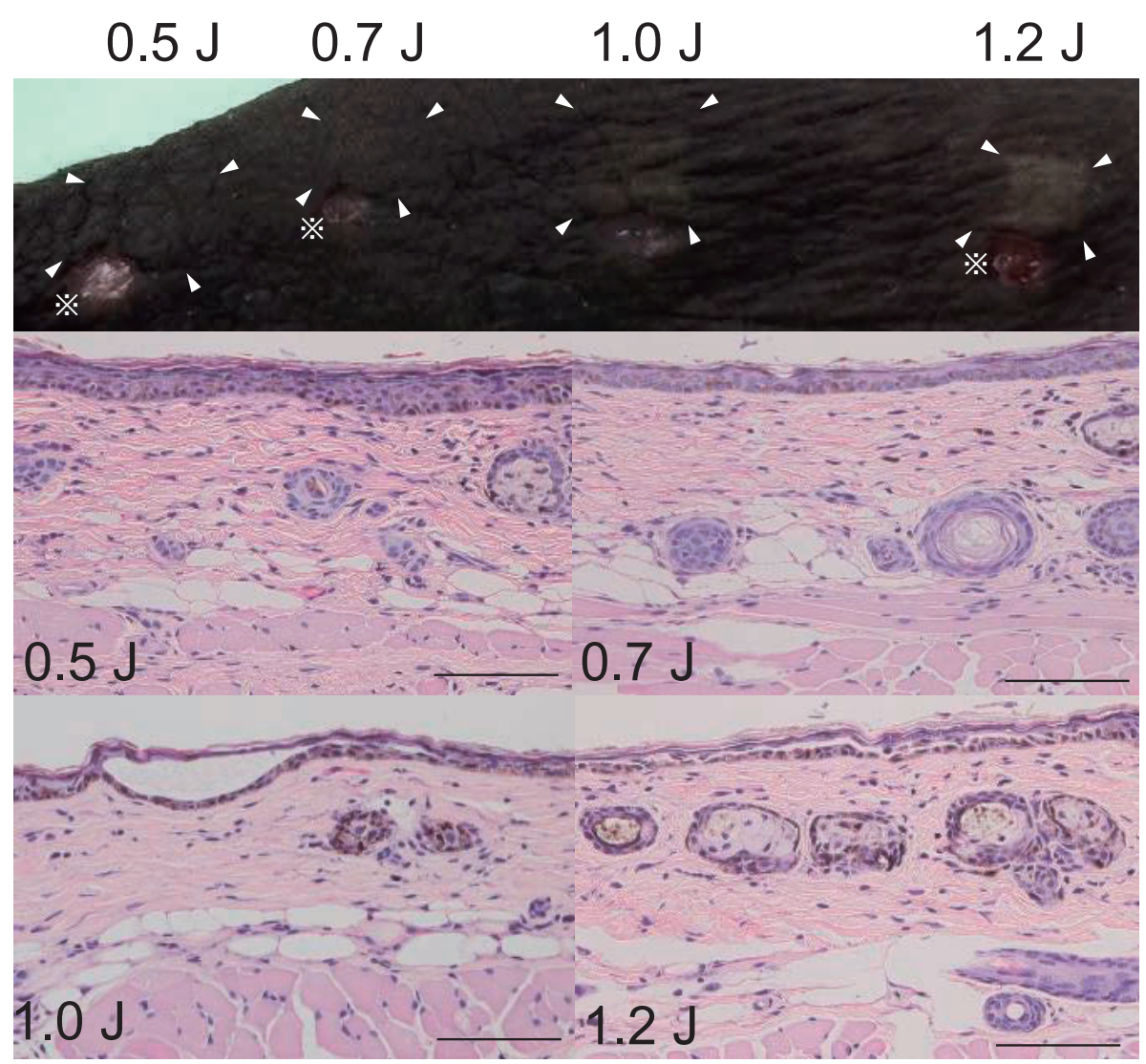

Fig. 1 The effect of a frequency-doubled Nd:YAG laser on a BHSCF (black hairless SCF) mouse. Upper column: representative photograph of the mouse just after laser irradiation. Second and third column: histology of the skin taken $1 \mathrm{~min}$ after laser irradiation.

Laser irradiation at $1.0 \mathrm{~J} / \mathrm{cm}^{2}$ and $1.2 \mathrm{~J} / \mathrm{cm}^{2}$ clearly shows whitening of the skin. Vacuolar formations and partial separation of the epidermis and dermis were seen in the irradiated sites of $1.0 \mathrm{~J} / \mathrm{cm}^{2}$ and $1.2 \mathrm{~J} / \mathrm{cm}^{2}$. The arrow indicates the irradiated area. The whitish mark near the laser-irradiated area is the marking to know the precise area of the laser irradiation. Scale bar, $100 \mu \mathrm{m}$.

\begin{abstract}
Materials and Methods
Mice

Hairless mice harboring melanocytes in the epidermis, containing only eumelanin, mainly pheomelanin with much less eumelanin, or no melanin, were generated as described previously ${ }^{4}$. Briefly, K14-stem cell factor (SCF) transgenic mice ${ }^{5}$ were crossed with yellow mice which have the recessive yellow allele due to a frameshift causing a prematurely terminated nonfunctioning melanocortin 1 receptor (MC1R) (obtained from the Jackson Laboratory) $^{6}$ to produce black (termed BSCF: black SCF) and yellow (termed YSCF: yellow SCF) mice with epidermal melanocytes as keratinocyte-derived SCF can maintain melanocytes in the epidermis. Hairless albino HOShr-1 mice (purchased from Japan SLC, Inc. Shizuoka, Japan) were crossed with BSCF and YSCF mice to produce hairless mice with epidermal melanocytes which synthe-
\end{abstract}

size only eumelanin (BHSCF: black hairless SCF), dominantly pheomelanin (YHSCF: yellow hairless SCF), and no melanin (WHSCF: white hairless SCF).

The melanin content of these mice was previously reported $^{4}$. Briefly, the amount of eumelanin was evaluated by assaying the specific degradation product, pyrrole2,3,5-tricarboxylic acid (PTCA) $)^{7,8}$ and the amount of pheomelanin was evaluated by the amount of 4-amino-3hydroxyphenylalanine (4-AHP) ${ }^{9}$. One nanogram of PTCA or 4-AHP corresponds to $25 \mathrm{ng}$ of eumelanin or $9 \mathrm{ng}$ of pheomelanin, respectively ${ }^{8,9}$. The average amount of PTCA and 4-AHP was $1,373 \mathrm{ng} / \mathrm{mg}$ and $1.3 \mathrm{ng} / \mathrm{mg}$ in BHSCF, $73.8 \mathrm{ng} / \mathrm{mg}$ and $402 \mathrm{ng} / \mathrm{mg}$ in YHSCF mice, and $<1 \mathrm{ng} / \mathrm{mg}$ and $1.7 \mathrm{ng} / \mathrm{mg}$ in WHSCF mice ${ }^{4}$.

These mice were fed on a standard diet and water, and supported at a controlled temperature and humidity with a $12 \mathrm{~h}$ light/dark cycle in our University Animal Facility. 


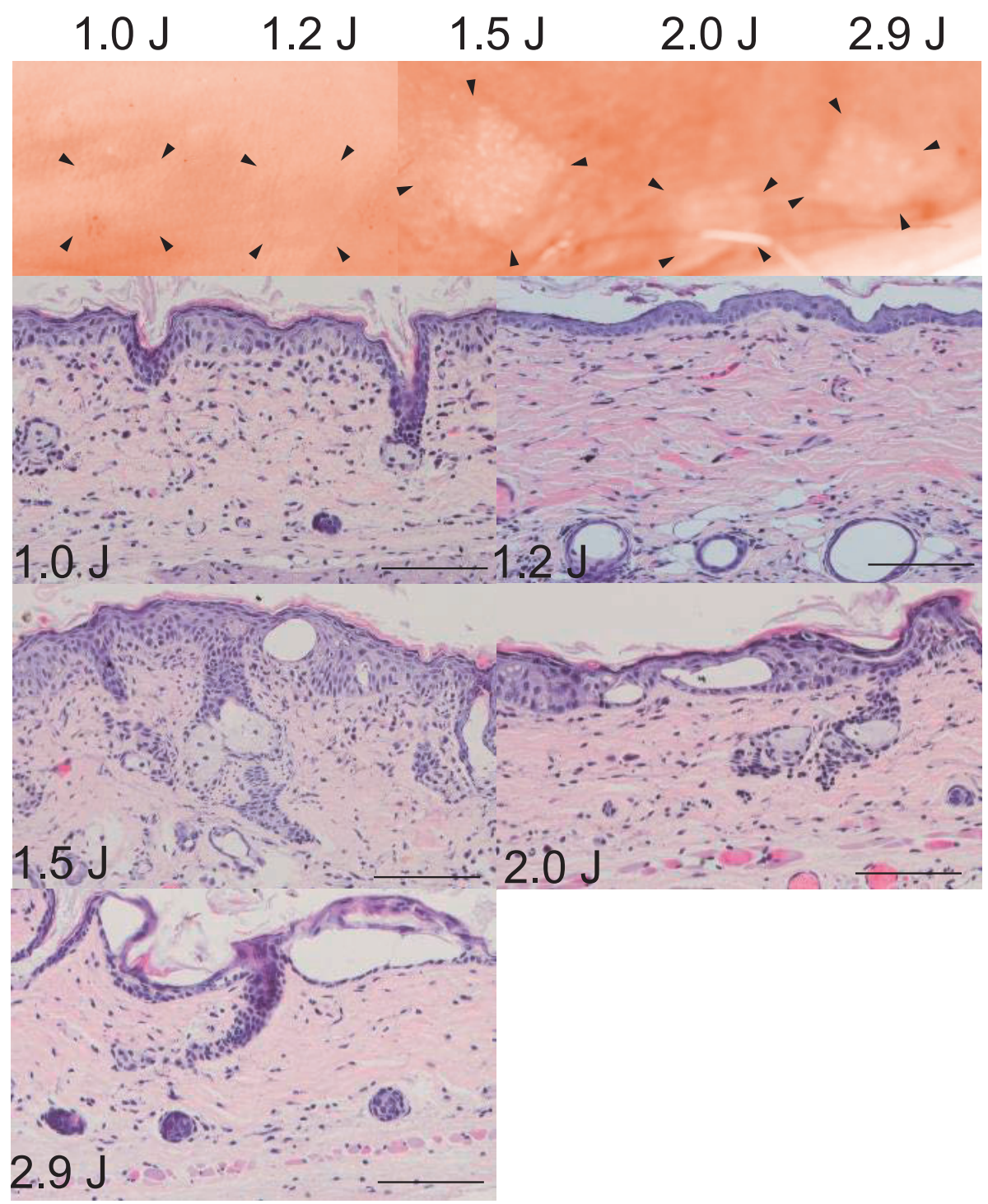

Fig. 2 The effect of a frequency-doubled Nd:YAG laser on a YHSCF (yellow hairless SCF) mouse. Upper column: representative photograph of the mouse just after laser irradiation. Second, third, and forth column: histology of the skin taken $1 \mathrm{~min}$ after laser irradiation.

Laser irradiation at $1.5 \mathrm{~J} / \mathrm{cm}^{2}, 2.0 \mathrm{~J} / \mathrm{cm}^{2}$, and $2.9 \mathrm{~J} / \mathrm{cm}^{2}$ clearly shows whitening of the skin. Vacuolar formations and partial separation of the epidermis and dermis were seen in the irradiated sites of $1.5 \mathrm{~J} / \mathrm{cm}^{2}, 2.0 \mathrm{~J} / \mathrm{cm}^{2}$, and $2.9 \mathrm{~J} / \mathrm{cm}^{2}$. The arrow indicates the irradiated area. Scale bar, $100 \mu \mathrm{m}$.

This study was approved by the Institutional Animal Care and Use Committee and carried out according to the Nippon Medical School Animal Experimentation Regulations.

\section{Irradiation of Mice}

A Q-switched frequency-doubled Nd:YAG laser (myQ: dual; Cutera Inc., Brisbane, CA) with the following settings: $532 \mathrm{~nm}, 6 \mathrm{~ns}$, spot size of $4 \times 4 \mathrm{~mm}$ (square) was used for irradiation. Each mouse was irradiated at $0.5 \mathrm{~J} /$ $\mathrm{cm}^{2}, 0.7 \mathrm{~J} / \mathrm{cm}^{2}, 1.0 \mathrm{~J} / \mathrm{cm}^{2}$, and $1.2 \mathrm{~J} / \mathrm{cm}^{2}$ on the dorsal skin in the first set, and $0.4 \mathrm{~J} / \mathrm{cm}^{2}, 0.8 \mathrm{~J} / \mathrm{cm}^{2}, 1.0 \mathrm{~J} / \mathrm{cm}^{2}$, and
$1.2 \mathrm{~J} / \mathrm{cm}^{2}$ in BHSCF mice and $1.0 \mathrm{~J} / \mathrm{cm}^{2}, 1.5 \mathrm{~J} / \mathrm{cm}^{2}, 2.0 \mathrm{~J} /$ $\mathrm{cm}^{2}$, and $2.9 \mathrm{~J} / \mathrm{cm}^{2}$ in YHSCF and WHSCF mice in the second set. Each group in the experiment consisted of 3 mice of each skin color. Skin specimens were taken 1 minute after each laser irradiation.

\section{Histological Analysis}

Biopsied skin was fixed in $4 \%$ buffered paraformaldehyde, embedded in paraffin, stained using hematoxylin and eosin, and observed under light microscopy. 


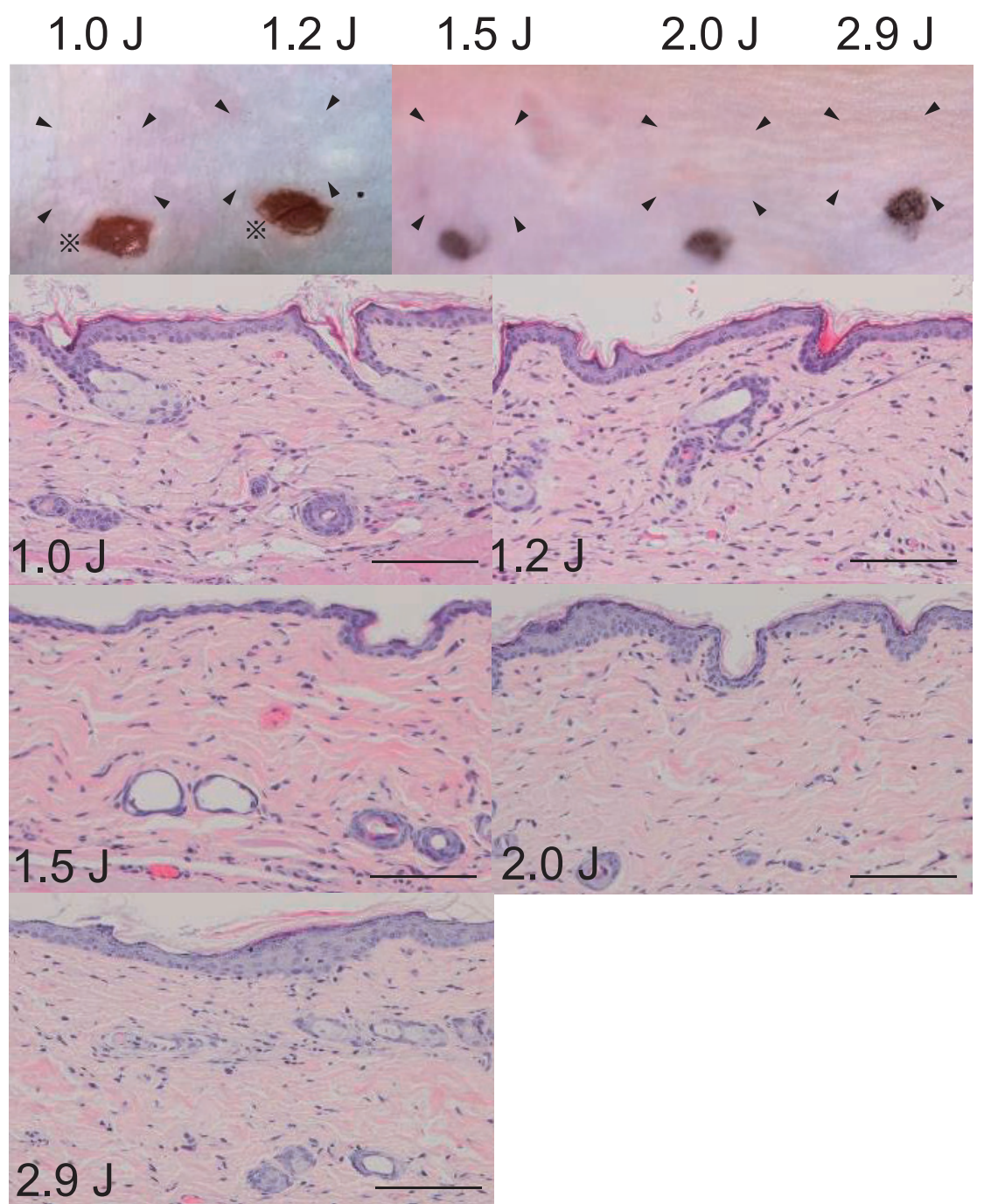

Fig. 3 The effect of a frequency-doubled Nd:YAG laser on a WHSCF (white hairless SCF) mouse. Upper column: representative photograph of the mouse just after laser irradiation. Second, third, and forth column: histology of the skin taken $1 \mathrm{~min}$ after laser irradiation.

Laser irradiation at any fluence, including $2.9 \mathrm{~J} / \mathrm{cm}^{2}$ (maximum fluence), did not induce whitening of the skin or any vacuolar formation. The arrow indicates the irradiated area. The black mark near the laser-irradiated area is the marking to know the precise area of laser irradiation. Scale bar, $100 \mu \mathrm{m}$.

\section{Results}

Effect of a Q-switched Frequency-doubled Nd:YAG laser on BHSCF, YHSCF, and WHSCF Mice

As was previously reported, irradiation using a Qswitched laser on pigmented skin causes explosive vaporization of melanosomes which causes a temporary whitening of the skin due to microcavity formation ${ }^{10}$. This whitening of the skin was observed by irradiation of $1.0 \mathrm{~J} / \mathrm{cm}^{2}$ and $1.2 \mathrm{~J} / \mathrm{cm}^{2}$ in BHSCF mice (Fig. 1), and 1.5 $\mathrm{J} / \mathrm{cm}^{2}, 2.0 \mathrm{~J} / \mathrm{cm}^{2}$, and $2.9 \mathrm{~J} / \mathrm{cm}^{2}$ in YHSCF mice (Fig. 2). However, in WHSCF mice, irradiation at $2.9 \mathrm{~J} / \mathrm{cm}^{2}$ (maximum fluence) did not induce whitening of the skin (Fig. 3). These reactions were similarly observed in all mice.
Vacuolar formations and partial separation of the epidermis and dermis were seen only in the skin where a whitening change was observed (Fig. 1 3). This is compatible with the previous reports that heat generated by laser induced cavity formation results in temporary whitening of the skin ${ }^{11}$. Furthermore, this is melanin specific, as WHSCF which has no melanin showed no such changes.

\section{Discussion}

When irradiating skin by a laser with a wavelength that is absorbed by melanin, it is well known that darker skin types should be irradiated with a lower fluence to avoid 
burns. For this purpose, Fitzpatrick's phototype classification is extensively used ${ }^{12}$. However, as this classification is based on self-reported erythema sensitivity and tanning ability, there is limitation in its reliability. The effect of a laser on melanin has been well studied using extracted or synthetic melanin, therefore the effect of a laser needs to be considered by the content of melanin in the skin. Our results showed that yellow mice which contained dominantly pheomelanin in the epidermis were resistant to heat degeneration by a frequency-doubled Nd:YAG laser compared to black mice which have only eumelanin. As optical absorption of red hair has been reported to be lower compared to that of black hair, thermal injury might be less in the pheomelanin-containing cells compared with that in the eumelanin-containing cells ${ }^{13}$.

Recently, individual typology angle (ITA) by image analysis and total melanin content were reported to be well correlated, especially with PTCA (the degradation product of DHICA melanin) and thiazole-2,4,5tricarboxylic acid (the degradation product of benzothiazine-type pheomelanin $)^{14}$. As melanin plays a key role in a laser's effect, skin color evaluation by image analysis such as ITA will offer better information before laser treatment and our results showed the different thermal effect in the skin with different melanin subspecies.

In summary, we showed the different heat degeneration by a frequency-doubled Nd:YAG laser using mice containing dominantly pheomelanin or eumelanin. This phenomana is reproducibly observed in these mice, which indicates that melanin type and content plays a major role in heat degeneration in mice. This might be due to these mice having the advantage of a uniform background melanin content. A precise definition of skin color and a characterization of the melanin chemical phenotype might be needed to better understand the laser effect in the future.

Acknowledgements: The authors appreciate Mrs. Fumino Oda for her technical help in this experiment.

Conflict of Interest: The Q-switched frequency-doubled Nd: YAG laser was lent by Cutera Inc.

\section{References}

1. Ito S, Wakamatsu K: Human hair melanins: what we have learned and have not learned from mouse coat color pigmentation. Pigment Cell Melanoma Res 2011; 24: 63-
74.

2. Wakamatsu K, Ohtara K, Ito S: Chemical analysis of late stages of pheomelanogenesis: conversion of dihydrobenzothiazine to a benzothiazole structure. Pigment Cell Melanoma Res 2009; 22: 474-486.

3. Crippa PR, Cristofoletti V, Romeo N: A band model for melanin deducted from optical absorption and photoconductivity experiments. Biochim Biophys Acta 1978; 538: 164-170.

4. Okazaki S, Funasaka Y, Wakamatsu K, Kawana S, Saeki $\mathrm{H}$ : Effect of infrared radiation $\mathrm{A}$ on photoaged hairless mice harboring eumelanin and pheomelanin in the epidermis. J Dermatol 2015; 42: 382-390.

5. Kunisada T, Lu SZ, Yoshida H, Nishikawa S, Nishikawa S, Mizoguchi M, Hayashi S, Tyrrell L, Williams DA, Wang $\mathrm{X}$, Longley BJ: Murine cutaneous mastocytosis and epidermal melanocytosis induced by keratinocyte expression of transgenic stem cell factor. J Exp Med 1998; 187: 15651573.

6. Robbins LS, Nadeau JH, Johnson KR, Kelly MA, RoselliRehfuss L, Baack E, Mountjoy KG, Cone RD: Pigmentation phenotypes of variant extension locus alleles result from point mutations that alter $\mathrm{MSH}$ receptor function. Cell 1993; 72: 827-834.

7. Ito S, Fujita K: Microanalysis of eumelanin and pheomelanin in hair and melanomas by chemical degradation and liquid chromatography. Anal Biochem 1985; 144: 527536.

8. Ito S, Nakanishi Y, Valenzuela RK, Brilliant MH, Kolbe L, Wakamatsu K: Usefulness of alkaline hydrogen peroxide oxidation to analyze eumelanin and pheomelanin in various tissue samples: application to chemical analysis of human hair melanins. Pigment Cell Melanoma Res 2011; 24: 605-613.

9. Wakamatsu K, Ito S, Rees JL: The usefulness of 4-amino3-hydroxyphenylalanine as a specific marker of pheomelanin. Pigment Cell Res 2002; 15: 225-232.

10. Hruza JG, Dover JS, Flotte TJ, Goetschkes M, Watanabe S, Anderson RR: Q-switched ruby laser irradiation of normal human skin. Histologic and ultrastructural findings. Arch Dermatol 1991; 127: 1799-1805.

11. Negishi K, Akita H, Matsunaga Y: Prospective study of removing solar lentigines in Asians using a novel dualwavelength and dual-pulse width picosecond laser. Lasers Surg Med 2018; 50: 851-858.

12. Fitzpatrick TB: The validity and practicality of sunreactive skin types I through VI. Arch Dermatol 1988; 124: 869-871.

13. Menon IA, Persad S, Haberman HF, Kurian CJ: A comparative study of the physical and chemical properties of melanins isolated from human black and red hair. J Invest Dermatol 1983; 80: 202-206.

14. Del Bino S, Ito S, Sok J, Nakanishi Y, Bastien P, Wakamatsu K, Bernerd F: Chemical analysis of constitutive pigmentation of human epidermis reveals constant eumelanin to pheomelanin ratio. Pigment Cell Melanoma Res 2015; 28: 707-717. 